Tropical Journal of Pharmaceutical Research September 2014; 13 (9): 1385-1391

ISSN: $1596-5996$ (print); 1596-9827 (electronic)

(c) Pharmacotherapy Group, Faculty of Pharmacy, University of Benin, Benin City, 300001 Nigeria.

All rights reserved.

Available online at http://www.tjpr.org

Original Research Article

http://dx.doi.org/10.4314/tjpr.v13i9.2

\title{
Evaluation of Hydrogels Based on Poloxamer 407 and Polyacrylic Acids for Enhanced Topical Activity of Gentamicin against Susceptible Infections
}

\author{
PO Nnamani, FC Kenechukwu*, CL Anugwolu and AA Attama \\ Drug Delivery Research Unit, Department of Pharmaceutics, Faculty of Pharmaceutical Sciences, University of Nigeria, Nsukka \\ 410001, Enugu State, Nigeria
}

*For correspondence: Email: chimafrankduff@yahoo.com; frankline.kenechukwu@unn.edu.ng

Received: 23 July 2013

Revised accepted: 20 July 2014

\begin{abstract}
Purpose: To formulate hydrogels based on poloxamer 407 and polyacrylic acids (Carbopols $\AA$ 971P and 974P), and evaluate its suitability for enhanced topical delivery of gentamicin, a potent but highly toxic aminoglycoside antibiotic.

Methods: Topical hydrogels of gentamicin were produced using poloxamer 407 and polyacrlic acids (Carbopols ${ }^{\circledR}$ 971P and 974P), and evaluated in terms of drug content, pH, physical assessment, viscosity and stability. In vitro antimicrobial drug release was conducted using four microorganisms Escherichia coli, Salmonella typhi, Staphylococcus aureus and Pseudomonas aeruginosa.

Results: Stable gentamicin-loaded hydrogels with good encapsulation efficiency (maximum EE\% of 85.76 to $90.66 \%$ ) as well as greater zones of inhibition against all organisms (maximum range: $22.34 \pm$ 2.35 to $29.40 \pm 3.07 \mathrm{~mm}$ ) than commercially available gentamicin ointment and pure sample of gentamicin $(p<0.05)$, were produced. Overall, poloxamer 407 hydrogels of gentamicin gave the most desirable properties in terms of $\mathrm{pH}$, viscosity and rapid concentration and time-dependent antibacterial activity on all tested micro-organisms, superior to polyacrylic acids hydrogels of gentamicin.

Conclusion: This study demonstrates that poloxamer 407 hydrogels of gentamicin may offer a promising approach for topical delivery of gentamicin for the treatment of skin infections caused by gentamicin-susceptible bacteria.
\end{abstract}

Keywords: Hydrogels, Gentamicin, Polyacrylic acid, Viscosity, Bioactivity, Poloxamer 407

Tropical Journal of Pharmaceutical Research is indexed by Science Citation Index (SciSearch), Scopus, International Pharmaceutical Abstract, Chemical Abstracts, Embase, Index Copernicus, EBSCO, African Index Medicus, JournalSeek, Journal Citation Reports/Science Edition, Directory of Open Access Journals (DOAJ), African Journal Online, Bioline International, Open-J-Gate and Pharmacy Abstracts

\section{INTRODUCTION}

Hydrogels, swollen three-dimensional networks of hydrophilic polymers held together by association bonds or cohesive forces, are suitable carriers for drug delivery [1]. They have high water content and rubbery nature similar to natural tissue, which make them desirable for biomedical applications [2-8]. In this type of device, a drug is incorporated into the system and then released in response to a change in the environment. The polymeric biomaterials are employed in hydrogel formulations to delay the dissolution of drug depending on the exposure of drug molecules to aqueous environment surrounding the drug delivery system. Their usage is advantageous in safety, ease of manufacture, cost effectiveness, biocompatibility and biodegradability [4]. In general, the gelation of a polymeric solution can be triggered by variations of temperature, as for poloxamers [9] or $\mathrm{pH}$, as for carbopols $[10,11]$. 
Gentamicin sulphate is an aminoglycoside antibiotic commonly used in the control of severe Gram positive and Gram negative microbial infections especially in burns and wounds as well as for treating bone and soft tissue infections [2]. Despite its benefits, bacterial barriers and adverse effects such as nephrotoxicity, ototoxicity and neurotoxicity upon prolonged use limit gentamicin daily dosage [7].

Formulation efforts, including use of hydrogels among others, have been made to determine its optimal therapeutic regimens in order to increase its overall efficacy while minimizing drug toxicity [6-8]. Topical hydrogels could be employed as an alternative low dose regimen aimed not only at reducing the limitations of toxicity associated with prolonged use of gentamicin but also assuring proper utilization of the benefits of gentamicin and its rapid bactericidal activity, especially in blood stream infections. Thus, this work seeks to design a gentamicin-loaded topical hydrogel and evaluate its antimicrobial properties against four micro-organisms (Escherichia coli, Bacillus subtilis, Staphylococcus aureus and Pseudomonas aeruginosa).

Consequently, the objective of the present study was to assess the topical delivery of gentamicin using polymeric hydrogels, and to determine the resultant bioactivity of the drug.

\section{EXPERIMENTAL}

\section{Materials}

Gentamicin pure power (a kind gift from JUHEL Pharmaceutical Limited, Awka, Nigeria), ethyl acetate, Ophthalidialdehyde (Sigma Aldrich, Germany), sodium chloride and sodium hydroxide (BDH, England), monobasic potassium phosphate (Sigma-Aldrich Chemical Co., USA), Carbopols ® 971P and 974P (The Lubrizol Corporation, USA), Poloxamer 407 (BASF Ludwigshafen, Germany) propylene glycol (Merck, Germany) and triethanolamine (Spectrum Chem. Mfg. Corp., California, USA) were used as procured from the manufacturers without further purification. All other reagents were analytical grade and used as such. Distilled water was obtained from an all-glass still. Clinical isolates of Staphylococcus aureus ATCC 13703 and Escherichia coli ATCC 9637 were obtained from Bishop Shanahan Hospital, Nsukka, Nigeria, whereas laboratory isolates of Bacillus subtilis and Pseudomonas aeruginosa were obtained from Department of Pharmaceutics, University of Nigeria, Nsukka, Nigeria.

\section{Preparation of gentamicin hydrogels}

Three gelling agents were employed poloxamer 407 (P407), and two polyacrylic acids (Cabopols $\AA$ 971P and 974P, i.e., C971 and C974). Poloxamer 407 was dissolved completely in purified water pre-cooled to approximately 5 ${ }^{\circ} \mathrm{C}$. Graded concentrations of gentamicin $(0.03$, 0.06 and $0.09 \mathrm{w} / \mathrm{w}$ ) were dissolved in aliquots of purified water and propylene glycol added, and mixed until a homogenous mass was formed. For the polyacrylic acid hydrogels, the same procedure was followed except that room temperature purified water was used instead of pre-cooled water and that the hydrogels were further neutralized by triethanolamine. All products were adjusted to $\mathrm{pH} 5.5$ and kept at room temperature for $24 \mathrm{~h}$ to ensure no entrapped air-bubble before they were dispensed in lacquered aluminum tube $(60 \mathrm{~g})$, securely closed and stored at room temperature until used. The formulation compositions are shown in Table 1.

\section{Physical examination and $\mathrm{pH}$ measurement}

The semi-solid formulations were physically examined for colour, homogeneity, and consistency. The $\mathrm{pH}$ was also re-evaluated (before each use) to make sure that it was stable within the skin $\mathrm{pH}$ of 5.5 [2].

\section{Drug content analysis}

About $0.5 \mathrm{~g}$ of hydrogel was dissolved in $10 \mathrm{ml}$ of water, centrifuged (TDL-4 B. Bran Scientific and Instru. Co., England) at $1000 \mathrm{rpm}$ for $30 \mathrm{~min}$, filtered through a Whatman No. 1 filter paper, adequately diluted and the concentration of gentamicin determined spectrophotometrically (Shimadzu UV-1601 UV/Vis double beam spectrophotometer, Japan) at $332 \mathrm{~nm}$ after derivatization with o-phthaldialdehyde reagent adopting Zhang's method [12].

\section{Rheological evaluation}

The viscosity assessment of the hydrogels was done using a Brookfield viscometer (GallenKamp, England). Due to the viscous nature of the formulations, 1,3 and $5 \mathrm{~g}$ quantities of the semi-solid formulations were dissolved in $25 \mathrm{ml}$ of purified water for $24 \mathrm{~h}$ and their viscosities determined.

\section{Determination of antimicrobial activity}

The antimicrobial activity of the hydrogels was tested against four microorganisms - Escherichia coli, Pseudomonas aeruginosa, Salmonella typhi 
and Staphylococus aureus by agar diffusion technique using samples withdrawn (at regular intervals) during the in vitro drug release studies (not shown). This method depends on the diffusion of antimicrobial agent from holes perforated in the microbe-seeded agar [13]. The agar plates were prepared following manufacturer's specifications and seeded with each bacterial isolate. The agar plates were allowed to set and a sterile cork borer $(8 \mathrm{~mm}$ diameter) was used to bore holes in the seeded agar medium. Using a sterile instrument, a definite volume withdrawn from the receptor compartment of the diffusion apparatus at predetermined time intervals was used to fill the holes. The plates were allowed to stand at room temperature for $15 \mathrm{~min}$ to enable prediffusion before incubating at $25 \pm 1^{\circ} \mathrm{C}$ for $48 \mathrm{~h}$. Triplicate tests were performed in each case. Growth was examined after incubation and the diameter of each inhibition zone was measured and the average determined.

\section{Stability study}

Stability study was carried out for the hydrogel formulations as per ICH guidelines [14] at $40^{\circ} \mathrm{C}$ in a humidity chamber having $75 \% \mathrm{RH}$ for 3 months, and thereafter withdrawn and drug content re-evaluated.

\section{Statistical analysis}

All experiments were performed in replicates for validity of statistical analysis. Results were expressed as mean \pm SD. ANOVA and Student's t-test were performed on the data sets generated using SPSS. Differences were considered significant for $p$-values $<0.05$.

\section{RESULTS}

\section{Physical evaluation and pH measurements}

All the hydrogels were transparent, clear and colourless. The Poloxamer 407 hydrogels were the most flexible and possessed the best physical assessment among the hydrogel formulations. Hydrogels of Carbopolß 974P were more consistent than those of Carbopol® $971 \mathrm{P}$. The $\mathrm{pH}$ of the hydrogels of Carbopols $\AA$ 971P and 974P before adjustment were more acidic than Poloxamer 407 that produced entirely basic hydrogels, hence the need to buffer the former with triethanolamine to a $\mathrm{pH}$ of 5.5 .

\section{Drug content analysis}

The results of the drug content analysis indicate that drug encapsulation efficiency (EE) increased with increase in the concentration of gentamicin up till $0.06 \% \mathrm{w} / \mathrm{w}$ for all batches, yielding maximum EE (\%) of 85.76, 93.00 and $90.66 \%$ for hydrogels formulated with P407, C971 and C974 respectively. Thereafter, the drug encapsulation efficiency decreased with increased drug loading. So, the hydrogels loaded with $0.06 \% \mathrm{w} / \mathrm{w}$ gentamicin resulted in higher EE $\%$ while those loaded with $0.09 \%$ w/w gentamicin gave the least.

\section{Viscosity measurement}

Results of the viscosity measurement (not shown) indicate that hydrogels of C974P (batches C0-C3) were the most viscous whereas hydrogels of P407 (batches A0-A3) were the least viscous among the polymeric hydrogels. The results equally revealed concentrationdependent increase in the viscosities of all the hydrogel formulations (i.e., $5 \mathrm{~g}>3 \mathrm{~g}>1 \mathrm{~g} / 25 \mathrm{ml}$ of distilled water). It was also observed that the drug-loaded hydrogels (batches A1-A3, B1-B3 and $\mathrm{C} 1-\mathrm{C} 3$ ) were more viscous than the plain hydrogels (batches $\mathrm{AO}, \mathrm{BO}$ and $\mathrm{C} 0$ ). Among the drug-loaded hydrogels viscosities decreased with increased drug loading irrespective of the polymeric hydrogel employed in the formulation, and as such hydrogel formulations loaded with

Table 1: Optimized formula for hydrogel preparations

\begin{tabular}{|c|c|c|c|c|c|}
\hline \multirow[t]{2}{*}{ Composition (g) } & \multicolumn{2}{|c|}{ Plain hydrogel (w/w) } & \multicolumn{3}{|c|}{ Drug-loaded hydrogel (w/w) } \\
\hline & $P 407$ & Carbopol $^{(B)}$ & 0.03 & 0.06 & 0.09 \\
\hline Poloxamer 407 & 13.2 & - & 13.2 & 13.2 & 13.2 \\
\hline Carbopol 971P & - & 5.0 & 5.0 & 5.0 & 5.0 \\
\hline Carbopol 974P & - & 5.0 & 5.0 & 5.0 & 5.0 \\
\hline Propylene glycol ${ }^{\text {(a) }}$ & 12.0 & - & 12.0 & 12.0 & 12 \\
\hline Propylene glycol ${ }^{(b)}$ & & 10.0 & 10 & 10 & 10.0 \\
\hline Triethanolamine $^{(\mathrm{b})}$ & - & Drop & Drop & Drop & Drop \\
\hline Purified water & 40.0 & 44.0 & q.s & q.s & q.s \\
\hline
\end{tabular}


whereas hydrogels loaded with $90 \mathrm{mg}$ of gentamicin (batches A3-C3) were the least viscous.

\section{In vitro antibacterial activity}

The results of drug release recorded as inhibitory zone diameter (IZD) are presented in Tables 2-6. The in vitro antibacterial activity of the formulations was compared with gentamicin pure sample and a commercially available gentamicin ointment. The results indicate that gentamicin loaded into the hydrogels produced very significant zones of inhibition against Gram positive organism ( $S$. aureus) and Gram negative organisms (S. typhi, E. coli and P. aeruginosa) used in the study.

\section{Stability of formulations}

The stability result (not shown) indicates insignificant difference in drug content of gentamicin before and after storage for 3 months. The result indicates that the formulations were stable at the required storage condition.

Table 2: Susceptibility of Escherichia coli to gentamicin in the hydrogel formulations

\begin{tabular}{lccccccc}
\hline Batch & code & \multicolumn{7}{c}{ Diameter of zone of inhibition $(\mathbf{m m})^{\mathrm{a}, \mathbf{b}}$} \\
\cline { 2 - 8 } & $\mathbf{7}$ & $\mathbf{2}$ & $\mathbf{3}$ & $\mathbf{4}$ & $\mathbf{5}$ & $\mathbf{6}$ & $\mathbf{7}$ \\
\cline { 2 - 8 } & $\mathbf{1}$ & & & & & \\
\hline $\mathrm{A}_{1}$ & $3.03 \pm 0.94$ & $6.18 \pm 1.02$ & $9.75 \pm 2.31$ & $12.46 \pm 1.98$ & $16.72 \pm 3.06$ & $19.09 \pm 2.84$ & $22.87 \pm 3.00$ \\
$\mathrm{~A}_{2}$ & $4.59 \pm 0.23$ & $7.73 \pm 0.94$ & $10.10 \pm 1.90$ & $13.89 \pm 2.09$ & $17.64 \pm 2.52$ & $21.47 \pm 1.88$ & $24.59 \pm 2.22$ \\
$\mathrm{~A}_{3}$ & $5.40 \pm 0.19$ & $8.28 \pm 0.08$ & $11.98 \pm 1.04$ & $14.76 \pm 1.22$ & $19.99 \pm 1.97$ & $24.00 \pm 2.55$ & $29.40 \pm 3.07$ \\
$\mathrm{~B}_{1}$ & $3.63 \pm 0.87$ & $5.82 \pm 0.59$ & $8.53 \pm 0.62$ & $11.95 \pm 0.89$ & $14.27 \pm 2.08$ & $17.14 \pm 3.00$ & $18.63 \pm 1.75$ \\
$\mathrm{~B}_{2}$ & $4.46 \pm 0.90$ & $6.37 \pm 0.67$ & $9.19 \pm 0.83$ & $12.44 \pm 2.00$ & $15.94 \pm 3.01$ & $18.86 \pm 1.98$ & $19.46 \pm 2.46$ \\
$\mathrm{~B}_{3}$ & $5.03 \pm 0.76$ & $8.16 \pm 0.45$ & $10.62 \pm 0.78$ & $13.95 \pm 0.83$ & $18.32 \pm 0.56$ & $23.18 \pm 2.14$ & $25.53 \pm 1.93$ \\
$\mathrm{C}_{1}$ & $2.18 \pm 0.07$ & $4.09 \pm 0.02$ & $6.00 \pm 0.17$ & $8.13 \pm 0.70$ & $10.25 \pm 1.96$ & $12.87 \pm 1.04$ & $13.72 \pm 0.99$ \\
$\mathrm{C}_{2}$ & $3.00 \pm 0.21$ & $5.32 \pm 0.45$ & $7.19 \pm 0.06$ & $9.08 \pm 0.14$ & $11.28 \pm 1.77$ & $13.64 \pm 2.05$ & $15.00 \pm 2.87$ \\
$\mathrm{C}_{3}$ & $4.13 \pm 0.94$ & $6.83 \pm 0.17$ & $8.08 \pm 0.74$ & $10.43 \pm 1.25$ & $12.93 \pm 0.82$ & $14.48 \pm 1.90$ & $17.30 \pm 1.56$ \\
$\mathrm{~S}_{1}$ & $2.98 \pm 0.07$ & $3.64 \pm 0.71$ & $4.99 \pm 0.01$ & $6.05 \pm 0.33$ & $8.19 \pm 0.15$ & $10.19 \pm 1.07$ & $12.47 \pm 1.88$ \\
$\mathrm{~S}_{2}$ & $2.00 \pm 0.16$ & $3.19 \pm 0.05$ & $4.07 \pm 0.09$ & $5.38 \pm 0.66$ & $6.77 \pm 0.90$ & $8.15 \pm 0.03$ & $10.98 \pm 2.07$ \\
\hline
\end{tabular}

${ }^{a}$ Mean $\pm S D,{ }^{b} n=3, A_{1}-A_{3}, B_{1}-B_{3}$, and $C_{1}-C_{3}$ are Poloxamer 407, Carbopol ${ }^{\otimes} 971 P$ and Carbopol $974 P$ hydrogels containing 30,60 and $90 \mathrm{mg}$ of gentamicin sulphate respectively; $S_{1}$ and $S_{2}$ are commercial gentamicin ointment and pure sample of gentamicin, respectively

Table 3: Susceptibility of Staphylococcus aureus to gentamicin in the hydrogel formulations

\begin{tabular}{|c|c|c|c|c|c|c|c|}
\hline \multirow{3}{*}{$\begin{array}{l}\text { Batch } \\
\text { code }\end{array}$} & \multicolumn{7}{|c|}{ Diameter of zone of inhibition $(\mathrm{mm})^{\mathrm{a}, \mathrm{b}}$} \\
\hline & \multicolumn{7}{|c|}{ Time (h) } \\
\hline & 1 & 2 & 3 & 4 & 5 & 6 & 7 \\
\hline$A_{1}$ & $2.99 \pm 0.45$ & $4.59 \pm 1.67$ & $7.43 \pm 2.20$ & $10.96 \pm 1.79$ & $13.82 \pm 3.10$ & $17.19 \pm 2.48$ & $20.22 \pm 3.93$ \\
\hline$A_{2}$ & $4.03 \pm 0.78$ & $7.29 \pm 1.09$ & $11.80 \pm 1.33$ & $15.24 \pm 2.05$ & $18.07 \pm 2.41$ & $21.26 \pm 0.99$ & $23.87 \pm 1.56$ \\
\hline$A_{3}$ & $5.00 \pm 0.82$ & $8.57 \pm 1.46$ & $12.23 \pm 1.00$ & $16.04 \pm 1.72$ & $19.68 \pm 2.15$ & $23.80 \pm 2.75$ & $27.49 \pm 2.38$ \\
\hline $\mathrm{B}_{1}$ & $2.96 \pm 0.09$ & $4.37 \pm 0.08$ & $7.23 \pm 0.19$ & $10.19 \pm 1.04$ & $13.62 \pm 1.53$ & $16.89 \pm 1.44$ & $17.32 \pm 1.09$ \\
\hline $\mathrm{B}_{2}$ & $4.02 \pm 0.50$ & $5.98 \pm 0.17$ & $8.67 \pm 0.25$ & $11.87 \pm 1.23$ & $14.63 \pm 1.09$ & $17.95 \pm 1.18$ & $18.64 \pm 1.33$ \\
\hline $\mathrm{B}_{3}$ & $4.95 \pm 0.67$ & $6.62 \pm 0.88$ & $9.03 \pm 0.72$ & $12.83 \pm 0.98$ & $17.95 \pm 1.82$ & $22.56 \pm 2.07$ & $24.35 \pm 1.98$ \\
\hline $\mathrm{C}_{1}$ & $2.04 \pm 0.19$ & $3.89 \pm 0.12$ & $5.47 \pm 0.11$ & $6.78 \pm 0.54$ & $8.15 \pm 0.96$ & $10.37 \pm 1.45$ & $11.72 \pm 1.69$ \\
\hline $\mathrm{C}_{2}$ & $2.95 \pm 0.43$ & $4.07 \pm 0.35$ & $6.55 \pm 0.32$ & $7.83 \pm 0.09$ & $9.74 \pm 0.85$ & $11.91 \pm 1.73$ & $13.53 \pm 1.04$ \\
\hline $\mathrm{C}_{3}$ & $3.68 \pm 0.81$ & $5.97 \pm 0.06$ & $7.66 \pm 0.94$ & $9.34 \pm 0.87$ & $11.52 \pm 1.06$ & $13.81 \pm 1.75$ & $16.56 \pm 1.50$ \\
\hline $\mathrm{S}_{1}$ & $2.02 \pm 0.98$ & $2.46 \pm 0.74$ & $3.71 \pm 0.19$ & $5.64 \pm 0.43$ & $7.98 \pm 0.95$ & $9.16 \pm 0.79$ & $11.87 \pm 1.47$ \\
\hline $\mathrm{S}_{2}$ & $1.98 \pm 0.62$ & $2.28 \pm 0.39$ & $3.59 \pm 0.97$ & $4.77 \pm 0.56$ & $5.14 \pm 0.79$ & $7.81 \pm 0.35$ & $9.77 \pm 0.72$ \\
\hline
\end{tabular}


Table 4: Susceptibility of Salmonella typhi to gentamicin in the hydrogel formulations

\begin{tabular}{|c|c|c|c|c|c|c|c|}
\hline \multirow{3}{*}{$\begin{array}{l}\text { Batch } \\
\text { code }\end{array}$} & \multicolumn{7}{|c|}{ Diameter of zone of inhibition $(\mathrm{mm})^{\mathrm{a}, \mathrm{b}}$} \\
\hline & \multicolumn{7}{|c|}{ Time (h) } \\
\hline & 1 & 2 & 3 & 4 & 5 & 6 & 7 \\
\hline$\overline{A_{1}}$ & $2.00 \pm 0.91$ & $4.69 \pm 0.83$ & $6.78 \pm 0.52$ & $9.05 \pm 0.14$ & $12.43 \pm 1.49$ & $15.17 \pm 2.33$ & $17.68 \pm 2.38$ \\
\hline $\mathrm{A}_{2}$ & $3.25 \pm 0.66$ & $5.14 \pm 0.26$ & $7.90 \pm 0.63$ & $10.26 \pm 1.08$ & $13.65 \pm 1.17$ & $16.74 \pm 1.20$ & $20.23 \pm 2.19$ \\
\hline$A_{3}$ & $4.16 \pm 0.57$ & $6.05 \pm 0.81$ & $8.13 \pm 0.67$ & $11.27 \pm 1.09$ & $14.80 \pm 1.25$ & $18.99 \pm 1.04$ & $22.34 \pm 2.35$ \\
\hline $\mathrm{B}_{1}$ & $1.95 \pm 0.08$ & $3.28 \pm 0.91$ & $5.23 \pm 0.09$ & $8.10 \pm 0.33$ & $10.26 \pm 1.74$ & $13.89 \pm 1.02$ & $15.94 \pm 1.66$ \\
\hline $\mathrm{B}_{2}$ & $2.66 \pm 0.14$ & $3.97 \pm 0.65$ & $5.76 \pm 0.65$ & $8.87 \pm 0.24$ & $11.63 \pm 1.08$ & $14.30 \pm 1.56$ & $16.91 \pm 1.07$ \\
\hline $\mathrm{B}_{3}$ & $3.28 \pm 0.25$ & $4.13 \pm 0.46$ & $6.35 \pm 0.72$ & $9.64 \pm 0.58$ & $12.81 \pm 1.40$ & $16.52 \pm 1.09$ & $20.43 \pm 1.48$ \\
\hline $\mathrm{C}_{1}$ & $1.83 \pm 0.90$ & $3.10 \pm 0.87$ & $4.26 \pm 0.76$ & $5.53 \pm 0.43$ & $6.45 \pm 0.12$ & $8.29 \pm 0.89$ & $9.76 \pm 0.97$ \\
\hline $\mathrm{C}_{2}$ & $2.32 \pm 0.17$ & $3.75 \pm 0.99$ & $4.70 \pm 0.38$ & $5.91 \pm 0.74$ & $7.82 \pm 0.58$ & $9.16 \pm 0.19$ & $11.80 \pm 1.22$ \\
\hline $\mathrm{C}_{3}$ & $3.07 \pm 0.61$ & $4.02 \pm 0.46$ & $5.89 \pm 0.77$ & $7.57 \pm 0.13$ & $9.24 \pm 0.34$ & $11.53 \pm 1.82$ & $14.86 \pm 1.90$ \\
\hline$S_{1}$ & $1.75 \pm 0.04$ & $2.00 \pm 0.64$ & $3.17 \pm 0.09$ & $4.74 \pm 0.35$ & $5.49 \pm 0.91$ & $7.82 \pm 1.17$ & $9.53 \pm 0.84$ \\
\hline $\mathrm{S}_{2}$ & $1.53 \pm 0.18$ & $1.87 \pm 0.19$ & $2.15 \pm 0.07$ & $3.24 \pm 0.63$ & $4.07 \pm 0.96$ & $5.98 \pm 0.15$ & $7.46 \pm 0.89$ \\
\hline
\end{tabular}

Table 5: Susceptibility of Pseudomonas aeruginosa to gentamicin in the hydrogel formulations

\begin{tabular}{|c|c|c|c|c|c|c|c|}
\hline \multirow[t]{2}{*}{$\begin{array}{l}\text { Batch } \\
\text { code }\end{array}$} & \multicolumn{7}{|c|}{$\begin{array}{c}\text { Diameter of zone of inhibition }(\mathrm{mm})^{\mathrm{a}, \mathrm{b}} \\
\text { Time }(\mathrm{h})\end{array}$} \\
\hline & 1 & 2 & 3 & 4 & 5 & 6 & 7 \\
\hline$A_{1}$ & $2.08 \pm 0.37$ & $4.57 \pm 0.28$ & $6.96 \pm 0.59$ & $9.15 \pm 0.72$ & $12.74 \pm 1.40$ & $15.89 \pm 1.48$ & $18.56 \pm 1.21$ \\
\hline$A_{2}$ & $3.76 \pm 0.25$ & $5.09 \pm 0.17$ & $7.78 \pm 0.91$ & $10.84 \pm 1.03$ & $13.50 \pm 1.06$ & $17.42 \pm 1.85$ & $21.39 \pm 2.58$ \\
\hline$A_{3}$ & $4.45 \pm 0.68$ & $6.67 \pm 0.22$ & $8.54 \pm 0.13$ & $11.98 \pm 0.77$ & $14.02 \pm 1.00$ & $18.19 \pm 1.56$ & $23.45 \pm 2.94$ \\
\hline$B_{1}$ & $2.00 \pm 0.19$ & $3.98 \pm 0.77$ & $6.15 \pm 0.47$ & $9.00 \pm 0.45$ & $11.47 \pm 0.99$ & $14.48 \pm 1.75$ & $16.98 \pm 1.55$ \\
\hline $\mathrm{B}_{2}$ & $3.54 \pm 0.82$ & $4.59 \pm 0.23$ & $6.62 \pm 0.98$ & $9.78 \pm 0.26$ & $12.03 \pm 1.14$ & $15.91 \pm 1.23$ & $17.49 \pm 1.09$ \\
\hline $\mathrm{B}_{3}$ & $4.20 \pm 0.76$ & $5.19 \pm 0.08$ & $7.98 \pm 0.56$ & $10.37 \pm 1.04$ & $13.25 \pm 1.06$ & $17.40 \pm 1.88$ & $21.35 \pm 1.78$ \\
\hline $\mathrm{C}_{1}$ & $1.92 \pm 0.08$ & $3.56 \pm 0.67$ & $4.80 \pm 0.19$ & $5.79 \pm 0.52$ & $7.62 \pm 0.71$ & $9.35 \pm 0.24$ & $10.19 \pm 1.06$ \\
\hline $\mathrm{C}_{2}$ & $2.53 \pm 0.17$ & $3.99 \pm 0.25$ & $5.18 \pm 0.70$ & $6.47 \pm 0.92$ & $8.25 \pm 0.38$ & $10.81 \pm 1.56$ & $12.74 \pm 1.87$ \\
\hline $\mathrm{C}_{3}$ & $3.13 \pm 0.09$ & $4.48 \pm 0.12$ & $6.72 \pm 0.36$ & $8.29 \pm 0.57$ & $10.34 \pm 1.02$ & $12.91 \pm 1.85$ & $15.68 \pm 0.50$ \\
\hline$S_{1}$ & $1.90 \pm 0.78$ & $2.09 \pm 0.47$ & $3.56 \pm 0.90$ & $4.45 \pm 0.35$ & $6.98 \pm 0.59$ & $8.71 \pm 0.97$ & $10.07 \pm 1.82$ \\
\hline $\mathrm{S}_{2}$ & $1.82 \pm 0.10$ & $1.97 \pm 0.59$ & $2.46 \pm 0.07$ & $3.67 \pm 0.38$ & $4.17 \pm 0.97$ & $6.33 \pm 0.35$ & $8.19 \pm 0.79$ \\
\hline
\end{tabular}

${ }^{a}$ Mean $\pm S D,{ }^{b} n=3, A_{1}-A_{3}, B_{1}-B_{3}$, and $C_{1}-C_{3}$ are Poloxamer 407, Carbopol ${ }^{\Theta} 971 P$ and Carbopol $974 P$ hydrogels containing 30, 60 and $90 \mathrm{mg}$ of gentamicin sulphate respectively; $S_{1}$ and $S_{2}$ are commercial gentamicin ointment and pure sample of gentamicin, respectively

\section{DISCUSSION}

The results of the physicochemical evaluation showed that the gentamicin-loaded hydrogels exhibited good physicochemical properties. The $\mathrm{pH}$ values of the gentamicin-loaded hydrogels would enhance the efficiency of the formulations as topical drug delivery systems for gentamicin as well as make the formulations safe for use on the skin, consistent with previous report [2]. The implication of the results of the drug content analysis is that gentamicin attained saturation solubility in the hydrogel matrices at $0.06 \% \mathrm{w} / \mathrm{w}$ loading for all batches, and so more of the drug could not be solubilized and encapsulated, consistent with earlier reports $[13,15]$. However, all the batches had good EE \%. Overall, batch B2 formulated with C971P and $0.06 \% \mathrm{w} / \mathrm{w}$ gentamicin gave the best drug content of 93.00 $\%$. Since gentamicin is freely soluble in water (hydrophilic), this result agrees with previous report that much of the drug was present in the aqueous phase of the formulations, loosely attached at or near the particle surface since the more hydrophilic the substance, the weaker the interaction with particle surface, and eventually the compound could be localized in the surfactant layer [16]. And when more drug particles at the periphery of the particle surface eventually encounter the polymeric cross-linked gel-matrices of polyacrylic acid resins and P407, stabilization would occur [17]. Thus, to improve the skin uptake and delivery of such a drug, a semi-solid vehicle such as topical hydrogels would be a better approach.

Results of the viscosity determination indicate that hydrogels of C974P (batches C0-C3) were the most viscous whereas hydrogels of P407 (batches A0-A3) were the least viscous among the polymeric hydrogels. This is justified since C974P which is a highly cross-linked polymer produced highly viscous gels with rheology 
similar to mayonnaise [10,11]. On the other hand, P407, being the least cross-linked polymer among the polymeric hydrogels [9] employed in this study produced the least viscous hydrogels. The increased viscosity of the drug-loaded hydrogels compared with the plain hydrogels may be related to the additional drug content in the formulation compositions of the former. Viscosity of the gel matrix is an important factor to consider in evaluation of drug penetration from gels across the skin or artificial membrane and is used to measure the extrudability of a gel [14]. The decreased viscosity of the hydrogels at high drug loadings (batches A2-C2 and A3-C3) not only implies that these hydrogel formulations would be easily extruded from their containers or packages but also indicates potential improvement in the diffusivity of gentamicin within the hydrogel network which in turn would facilitate flux, consistent with previous report [14]. The microbiological test was performed using samples withdrawn from the in vitro drug release studies to show an increasing IZD over time. Regarding the bioactivity of the formulations, previous studies indicate that gentamicin formulations had activity against $S$. aureus $[7,18,19]$, E. coli and S. typhi [13] and $P$. aeruginosa [2]. It was observed that the greater the amount of gentamicin loaded into the formulations, the greater the IZD produced and vice versa. The formulations thus exhibited capacity limited bioactivity. In addition, the release of gentamicin from the hydrogels depended on the compositions of the formulations since the results showed that batch A3 formulated with $\mathrm{P} 407$ and $90 \mathrm{mg}$ of gentamicin gave the greatest IZD against all the microorganisms compared with the rest of the formulations. Similarly, the antibacterial activity of the formulations was concentation and timedependent, manifested by an increasing IZD against the test organisms with time, which was produced by samples withdrawn over time during the course of the dissolution studies.

All batches of the hydrogels prepared with P407 (batches A1-A3) as well as all batches of C971Pbased hydrogel formulations (batches B1-B3) inhibited growth of all micro-organisms. In other words, these formulations released enough gentamicin that inhibited growth of all four organisms studied (Tables 7-10). High IZDs recorded against all test organisms within $1 \mathrm{~h}$ of the study especially with formulations based on P407 (batches A1-A3) was an indication that these formulations would have exhibited the fastest release of the entrapped drug, hence the fast antibacterial activities; whereas timedependent increases in IZDs within $7 \mathrm{~h}$ implies that these formulations had potentials for sustained drug release.

Moreover, all batches of the formulations gave greater zones of inhibition than the gentamicin pure sample as well as commercially available gentamicin ointment against all test organisms. However, hydrogels prepared from P407 and containing $90 \mathrm{mg}$ of gentamicin (batch A3) gave the greatest IZD against all test organisms. This formulation would be a useful alternative for enhanced delivery of gentamicin in the treatment of skin infections especially those caused by gentamicin-susceptible micro-organisms, thus encouraging further development of this formulation.

Assessment of the stability of novel formulations is always very important in drug product design and development. Stability could be viewed from the degradation of the active ingredients or physical property of the formulation [14]. In order to determine the change in drug content on storage, stability study was carried out on the gentamicin-loaded hydrogels before and after storage for three months. The result indicates that the formulations were stable at the required storage condition.

\section{CONCLUSION}

All the formulations show good drug encapsulation. Additionally, the bacterial isolates tested are susceptible to gentamicin incorporated into the hydrogels. In other words, the antibacterial activity of the drug was retained, with poloxamer 407 based-hydrogels exhibiting the best and fastest bioactivity. Thus, this delivery system may offer a promising approach for the treatment of topical infections caused by gentamicin-susceptible bacteria.

\section{ACKNOWLEDGEMENT}

The authors thank BASF, Ludwigshafen, Germany for the provision of Poloxamer 407 used in this study, and also acknowledge the support of the Lubrizol Corporation, USA for gift of Carbopols ${ }^{\circledR}$ 971P and 974P.

\section{REFERENCES}

1. Eljarrat-Binstock E, Raiskup F, Stepensky D, Domb AJ, Frucht-Pery J. Delivery of gentamicin to the rabbit eye by drug-loaded hydrogel iontophoresis. Invest Ophthalmol Visual Sci. 2004; 103: 1-12.

2. El-Gendy NA, Abdelbary GA, El-komy MH, Saafan AE. Design and evaluation of a bioadhesive patch for 
topical delivery of gentamicin sulphate. Curr Drug Deliv. 2009; 6: 50-57.

3. Stephens D, Kli L, Robinson D, Chen S, Chang HC, Liu RM, Tian $Y$, Ginsburg EJ, Gao X, Stultz $T$. Investigation of the in vitro release of gentamicin from a poly anhydride matrix. J Control Rel. 2000; 63: 305-17.

4. Mayol L, Quaglia F, Borzacchiello A, Ambrosio L, La Rotonda MI. A novel poloxamers/hyaluronic acid in situ forming hydrogel for drug delivery: Rheological, mucoadhesive and in vitro release properties. Eur $J$ Pharm Biopharm. 2008; 70: 199-206.

5. Raja RK, Abbulu K, Sudhakar M, Roopakarki S, Rajkumar B. Design and in vitro evaluation of modified release valsartan hydrogels. Int $J$ Drug Deliv. 2011; 3: 648-660.

6. Changez $M$, Burugapalli $K$, Koul V, Choudhary V. The effect of composition of poly (acrylic acid)- gelatin hydrogel on gentamicin sulphate release: in vitro. Biomaterials. 2003; 24: 527-536.

7. Ayhan F, Özkan S. Gentamicin release from photopolymerized PEG diacrylate and PHEMA hydrogel discs and their in vitro antimicrobial activities. Drug Deliv. 2007; 14: 433-439.

8. Sokmen N, Bican F, Ayhan F, Ayhan H. Chelating agent effect on the release of gentamicin from PEG-DA hydrogels. Hacette J Biol Chem. 2008; 36: 347-352.

9. Desai SD, Blanchard J. Evaluation of pluronic F127based sustained-release ocular delivery systems for pilocarpine using the albino rabbit eye model. J Pharm Sci. 1998; 10: 1190-1195.

10. Cho KH, Park Y-J, Choi H-G. Development of a sulglycotide-loaded oral gel with good stability and improved gastric ulcer inhibition. Drug Dev Res. 2012; 73: 325-331.
11. Srividya B, Cardoza RM, Amin PD. Sustained ophthalmic delivery of ofloxacin from a $\mathrm{pH}$ triggered in situ gelling system. J Control Rel. 2001; 2-3: 205-211.

12. Zhang $X$, Wyss UP, Pichora $D$, Goosen MFA. Biodegradable controlled antibiotic release devices for osteomyelitis: optimization of release properties. $J$ Pharm Pharmacol. 1994; 46: 718- 24.

13. Umeyor CE, Kenechukwu FC, Ogbonna JDN, Builders PF, Attama AA. Preliminary studies on the functional properties of gentamicin in SRMS-based solid lipid microparticles. Int J Novel Drug Deliv Tech. 2011; 1: 130-142.

14. Nnamani PO, Kenechukwu FC, Dibua EU, Ogbonna CC, Monemeh UL, Attama AA. Transdermal microgels of gentamicin. Eur J Pharm Biopharm. 2013; 84(2): 345 $-354$.

15. Umeyor CE, Kenechukwu FC, Ogbonna JDN, Chime SA, Attama AA. Preparation of novel solid lipid microparticles loaded with gentamicin and its evaluation in vitro and in vivo. J Microencapsul. 2012; 29: 296-307.

16. Küchler S, Abdel-Mottaleb M, Lamprecht A, Radowski $M R$, Haag $R$, Schäfer-Korting $M$. Influence of nanocarrier type and size on skin delivery of hydrophilic agents. Int J Pharm. 2009; 377: 169-172.

17. Souto EB, Doktorovova S, Boonme P. Lipid based colloidal systems (nanoparticles, microemulsions) for drug delivery to the skin: materials and end product formulation. J Drug Del Sci Tech. 2011; 21: 43-54.

18. Nishijima S, Kurokawa I. Antimicrobial resistance of Staphylococcus aureus isolated from skin infections. Int J Antimicrob Agents. 2002; 19: 241-3.

19. Ahangari A, Salouti $M$, Heidari Z, Kazemizadeh $A R$, Safari AA. Development of gentamicin-gold nanospheres for antimicrobial drug delivery to Staphylococcal infected foci. Drug Deliv. 2013; 20: 34-39. 